\title{
IV SYMPOSIUM INTERNACIONAL SOBRE EPH - GESTOSIS
}

\author{
Presentamos una información sobre el Symposium \\ celebrado en Herzliya, Israel, en Octubre de 1974.
}

Se discutieron aspectos epidemiológicos, patogénicos, diagnósticos y terapéuticos de la gestosis (toxemia).

Alrededor de treinta países estuvieron representados, por cerca de seiscientos participantes. El éxito de las deliberaciones fue propiciado por la eficiencia y la dedicación de los organizadores, especialmente de los miembros de la Profesión Médica y del Cuerpo Científico y Docente de Israel, así como por la hospitalidad fraternal por ellos prodigada, lo que dio a este certamen un ambiente de cordialidad y de exaltación de los valores humanos en la investigación médica.

Esta publicación es un homenaje a nuestros colegas de Israel y a las realizaciones espirituales, técnicas, científicas y sociales de ese país.

Los informes se basan en notas tomadas personalmente en las conferencias y discusiones. Con el fin de agrupar los temas lo más didácticamente posible, no se ha conservado estrictamente el orden cronológico de las presentaciones.

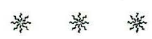

\section{Epidemiología}

En la comunicación "Perspectivas y engaños del estudio epidemiológico de la EPH Gestosis", R. F. Vollman (Ve- rikon, Suiza), define las bases para la aplicación de la epidemiología en la gestosis:

Desde las investigaciones sobre las enfermedades epidémicas, como el cólera, el tifus, la tuberculosis, la malaria, el bocio, el raquitismo, el beriberi y la desnutrición, se sabe que el curso, la duración, la gravedad y el desenlace de tales enfermedades pueden ser descritos cuantitativamente mediante la aplicación de ciertos métodos estadísticos. Lo que es más importante, por estos métodos se pueden someter a prueba hipótesis relativas a la etiología de las enfermeda des epidémicas. Asímismo, se pueden valorar los factores adyuvantes en la salud y la enfermedad, por ejemplo, edad, sexo, peso, nutrición, ocupación, clase social, educación, área geográfica, clima, condiciones estacionales. No obstante, la metodología epidemiológica, extensamente usada en otros campos, ha tenido escasa aplicación en Obstetricia y Ginecología.

Con respecto a la gestación, el autor señala lo siguiente:

1. En los embarazos normales las medianas de las presiones sanguíneas sistólica y diastólica tienen cursos similares: los valores más bajos se observan entre las semanas 20 y 27 ; entre las semanas 28 y 39 la presión su- 
be continuamente y alcanza el máximo al término.

2. No hay diferencias significativas en el curso de la presión sanguínea en embarazos no complicados, entre grávidas blancas y negras de clases económicas comparables.

3. Aparte de la edad y desde el comienzo hasta el final del embarazo, las primigrávidas tienen presiones más altas que las multigrávidas. Sinembargo en todos los grupos de paridad la presión aumenta consistentemente con la edad de la grávida.

4. La asociación de edema con el embarazo no eleva la presión mediana.

5. Las primigrávidas con proteinuria tienen presión terminal más alta que las primigrávidas normales.

6. No se observó edema ni proteinuria en 57 por ciento de grávidas blancas y en 69 por ciento de negras.

Finalmente el autor plantea la cuestión de si la EPH Gestosis es una unidad nosológica o una combinación arbitraria de procesos independientes.

Por su parte lan MacGillivray de la Universidad de Aberdeen ha estudiado "Los cambios que anteceden el comienzo clínico de la pre-eclampsia". Presenta sus observaciones en primigrávidas a las 30,34 y 38 semanas, con respecto al volumen del plasma, la concentración de proteínas del plasma, el agua corporal total, la excreción urinaria del estriol en 24 horas, la masa intravascular de proteínas, el sodio, el potasio y el cloro séricos. Este es un estudio prospectivo, encaminado a buscar los cambios posibles de aquellos parámetros en pacientes que eventualmente presentan toxemia.

A. Cárdenas Escobar (Bogotá, Colombia) presenta la "Incidencia de to- xemia - Factores socio-económicos". El status socio-económico es un factor significativo en la incidencia de toxemia del embarazo (pre-eclampsia y eclampsia), actuando probablemente a través de las condiciones nutricionales de la madre. Este es un hecho generalmente aceptado y ha sido mencionado por Mall-Haefeli (Basilea) en el II Symposium Internacional sobre Gestosis (Aarau, Suiza, Octubre de 1972).

La pobreza, la ignorancia y los horarios laborales inadecuados son causantes de la malnutrición prenatal, que lleva a tasas más elevadas de toxemia y de otras complicaciones del embarazo.

Los datos de este estudio destacan esta relación. Han sido recogidos en un lapso de once años (1961 a 1971) en una institución privada (Clínica de Maternidad David Restrepo, de Bogotá), fundación sin ánimo de lucro destinada a pacientes de la "clase media económica" (1), en su mayoría con educación secundaria. Durante este período fueron atendidos 28.201 casos obstétricos. De acuerdo con los criterios standard de diagnóstico (2), la tasa de toxemia en los once años fue de 3,4 por ciento, clasificada del modo siguiente:

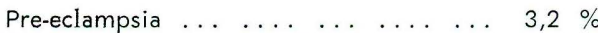

Pre-eclampsia asociada con

enfermedad hipertensiva $\ldots \begin{array}{lllll}\ldots & \ldots & \ldots & 0,1\end{array}$

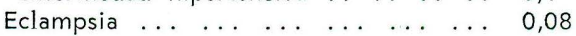

En un grupo socio-económico bajo tomado para comparación (Instituto Materno Infantil de Bogotá, 1968-72), la eclampsia ocurre con frecuencia

(1) Ingreso mensual aproximado de $\$ 2.500$ a $\$ 10.000$ pesos colombianos (US $\$ 80$ a $\$ 300$, ó 300 a 1.200 libras israelíes, en el momento de presentación de este estudio).

(2) Hipertensión (140/90 o superior) con edema $\mathrm{y} / \mathrm{o}$ proteinuria, después de la semana 24 de embarazo. 
diez veces mayor, o sea 0,8 por ciento.

También debe señalarse que la tasa de pre-eclampsia ha mostrado un descenso sugestivo en la Clínica David Restrepo entre los años 1965 y 1966, así :

Tasa hasta 1965, promedio .. . . . . $5,1 \%$ Tasa desde 1966, promedio ... ... . 2,4\%

A primera vista, no había explicación para esta reducción, puesto que no se habían introducido modificaciones en los criterios diagnósticos ni en los métodos de atención prenatal. Pero al hacer un análisis más detenido se observó que en 1965 se había creado una sección privada que implicó un cambio en la estructura socioeconómica del grupo atendido, en el sentido de la admisión de pacientes de una clase más alta con su propia tasa, más baja, de toxemia.

Las tasas observadas dentro de cada uno de estos componentes confirman esta impresión:

\begin{tabular}{lcl}
\hline & Pre-eclampsia & Eclampsia \\
$\begin{array}{c}\text { Toxemia en } \\
\text { pacientes afiliadas }\end{array}$ & $3,5 \%$ & $0,09 \%$ \\
$\begin{array}{c}\text { Toxemia en } \\
\text { pacientes privadas }\end{array}$ & $2,4 \%$ & $0,03 \%$ \\
\hline
\end{tabular}

A. Michael Davies, Rachel Poznansky, Rachel Prywes, Eliahu Sadovsky y Walter Czaczkes estudian la "Dieta y EPH Gestosis en Jerusalén". Han obtenido una historia dietética de 180 toxémicas y 360 controles normales, comparando cada toxémica con dos controles equiparables en cuanto a país de origen, paridad, mes probable del parto, edad, año de inmigración y nivel educacional. Concluyen que las diferencias en la dieta son efecto y no causa de la gestosis. Las pacientes con gestosis tuvieron una dieta pro- medio de 2.103 calorías, y los controles de 2.250 calorías.

\section{Patogenia}

En este capítulo predomina el tema de los trastornos de la coagulación y su significado en la gestosis.

Al respecto y bajo el título "Reto intelectual del problema de la coagulación de la sangre en el embarazo normal y patológico", el patólogo norteamericano Donald G. McKay hace las siguientes consideraciones:

1. Durante el embarazo normal hay un proceso "crónico" local de coagulación intravascular en los senos placentarios maternos, con depósitos de fibrina en los espacios intervellosos. El mecanismo que desencadena este fenómeno puede ser el contacto de la sangre con el trofoblasto alterado que contiene una gran cantidad de tromboplastina tisular. Esta coagulación local intravascular en la placenta produce cambios en la sangre circulante: aumento de los factores de coagulación (factores V, VII, VIII), de la fibrina soluble, de los productos de degradación de la fibrina, de la adherencia de las plaquetas y de la resistencia a la activación del sistema enzimático fibrinolítico. Así se constituye un estado de hipercoagulabilidad. En virtud de la superficie creciente de las vellosidades placentarias (sobre las cuales se deposita la fibrina), la coagulación aumenta gradualmente a medida que avanza el embarazo. Esto se expresa por el aumento progresivo de la fibrina soluble, de los productos de degradación y de la adherencia de las plaquetas.

2. Con la adición de la pre-eclampsia al embarazo, sobreviene una coagulación intravascular general y acelerada, cuyo mecanismo desencadenante es probablemente una actividad va- 
somotriz aumentada (vaso-espasmo). Este aparece en la microcirculación de muchos órganos. La generalización de la coagulación intravascular produce el depósito de fibrina macrocelular en la membrana basal y en las células endoteliales de los capilares glomerulares. El endotelio se hincha y disminuye la luz capilar; de aquí la albuminuria, la disminución de la filtración glomerular y la retención de sodio y otras substancias que debe eliminar el riñón.

3. Una aceleración súbita, "explosiva", del proceso lleva a la coagulación aguda, diseminada, masiva, intravascular, con aparición de trombos oclusivos formados por fibrina polimerizada y plaquetas en la microcirculación del cerebro, el hígado, las suprarrenales, los pulmones, los riñones, el tracto gastro-intestinal y el bazo. En estos órganos hay isquemia, hemorragia y necrosis, y clínicamente se presentan convulsiones, coma, shock, insuficiencia renal aguda, dolor abdominal, ictericia, hemólisis, edema y a veces desprendimiento de la retina. Aunque falta demostrar el mecanismo desencadenante de esta coagulación intravascular diseminada aguda de la eclampsia, una causa posible es el espasmo vascular severo y súbito, a su vez explicable por reactividad aumentada de la microcirculación. En resumen, el proceso se inicia con una coagulación intravascular crónica local, progresa hacia la coagulación crónica acelerada, aumentada, diseminada en la pre-eclampsia, y súbitamente se vuelve aguda, masiva, diseminada en la eclampsia.

H. Hoffbaver (Fravenklinik Charlottenburg der Freien Universitat, Berlin) en su comunicación "Alteración de la coagulación sanguínea en relación con depósitos anormales de fibrina en las placentas de gestosis", considera tres tipos de gestosis:
1. Gestosis con participación mayor del sistema vascular periférico, sin compromiso de la placenta.

2. Gestosis con localización principal en el área feto-materna, sin compromiso de la periferia.

3. Las formas combinadas y más graves de gestosis, que afectan la placenta y el sistema vascular periférico.

En los tipos 2 y 3 se encuentran depósitos de fibrina en la placenta, con reacción consecutiva del sistema periférico de coagulación.

La evidencia histológica de microfibrina inter y perivellosa se puede demostrar en las placentas de:

a) Recién nacidos eutróficos, maduros.

b) Hipotróficos ("small for da$\left.t e^{\prime \prime}\right)$.

c) Placentas de madres tratadas con heparina por retardo del crecimiento fetal. El tratamiento profiláctico con heparina permite reducir estos depósitos de fibrina y favorecer un desarrollo normal del feto, a pesar de la estructura anormal de las vellosidades.

S. Kunz, H. Weber y S. Heller, de la Universitats Frauenklinik, Tübingen, han hecho determinaciones hormonales y de los factores de coagulación en la gestosis ("Uber den Aussagewert hormoneller und gerinmungsphysiologischer Parameter bei der EPH-Gestose"). Estudiaron los siguientes parámetros en un grupo de 70 grávidas normales y en otro de 70 con EPH-Gestosis: recuento de plaquetas, fibrinógeno, antitrombina III, plasminógeno, inhibidores de plasmina y productos de degradación de la fibrina. Al mismo tiempo, y para evaluar el funcionamiento de la unidad feto-placentaria, se midieron por inmuno-ensayos en el suero y la orina 
la hormona lactogénica placentaria, la gonadotropina coriónica y el estriol.

Los resultados indicaron claramente en pacientes con gestosis una coagulación intravascular diseminada crónica, junto con función insuficiente de la unidad feto-placentaria.

El grupo de Tübingen ya había informado en el II Symposium (Aarau, Suiza, Octubre 1972) que "en las mujeres toxémicas tratadas con heparina se estimula a la vez la excreción de estriol (que se vuelve normal) y la secreción de hormona lactogénica placentaria" (Revista Ciba, Marzo 1973).

La hormona lactogénica placentaria humana (HLPH) se denomina también somatotrofina coriónica hum a $\mathrm{n}$ a $(\mathrm{SCH})$. Letchworth y Chard afirman que "con una técnica muy simple la obtención de tres o más valores inferiores a $4 \mathrm{mcg}$. por $\mathrm{ml}$. entre la 35 a y la 40: semanas del embarazo indican un riesgo de sufrimiento fetal o de asfixia neonatal del 70 por ciento". (Lancet, 1: 704, 1972).

Fritz K. Beller, de la Universidad de Muenster, Westfalia, República Federal Alemana ("The gestosis kidney, fact and fantasy"), estudiando biopsias renales con inmunofluoresceína y microscopía electrónica, en pacientes con gestosis, eclampsia y cuadros de coagulación intravascular diseminada y síndrome urémico, ha encontrado nefropatías diversas como glomerulonefritis, nefroesclerosis maligna y nefrosis; pero sólo raras veces la lesión glomerular descrita como típica de la toxemia. Los cuadros patológicos se encontraron incluso en pacientes sin antecedentes de enfermedad renal. Se observó igualmente que ellos pueden desarrollarse en el segundo trimestre del embarazo, sin proteinuria, edema ni hipertensión.
En los casos llamados "toxemia añadida a enfermedad renal" la biopsia mostró la afección original del riñón, sin lesión superpuesta típica de gestosis. Este punto, opuesto al concepto de McKay anteriormente relatado, fue motivo de controversia en el Symposium. Según Beller, el término "toxemia añadida" o "sobrepuesta" no encuentra justificación sobre bases morfológicas. Resulta también evidente que la agrupación de "gestosis" con base en la sintomatología clínica es engañosa. Se explican así la gran variedad y discrepancia de parámetros bioquímicos y otros, en este capítulo de la nosología.

El problema de la nefrosis es tratado por E. Rosenmann, N. Gilboa y A. Aviram, del Hospital Hadassah, Universidad Hebrea, Jerusalén ("Síndrome nefrótico y nefropatía pre-eclámptica").

En 10 años han observado solamente seis casos bien caracterizados de síndrome nefrótico en el embarazo con el cuadro de toxemia, que representan el 8 por ciento de todos los casos de nefrosis, y el 2 por ciento de todas las toxémicas hospitalizadas en ese período. La edad de las pacientes varió entre 18 y 26 años. En cuatro pacientes el síndrome nefrótico apareció en el 7\% mes de la primera gestación, y en dos al 8: mes de las gestaciones segunda y quinta. Todas presentaban edema severo. La presión sistólica varió entre 140 y 190 y la diastólica entre 100 y 120 . La eritrosedimentación estaba muy acelerada, por encima de $80 \mathrm{~mm}$. en la primera hora. La proteinuria era masiva, de 3 a $40 \mathrm{gm}$. en 24 horas. Los exámenes químicos de la sangre revelaron hipoalbuminemia $(1,1$ a $3,2 \mathrm{gm}$. por ciento), hipercolesterinemia (410 a 790 mg. por ciento) y aumento de los lípidos totales (855 a $1.300 \mathrm{mg}$. por ciento). La úrea sanguínea se encon- 
tró dentro de límites normales en 4 pacientes y ligeramente elevada en 2 (56 y 71 mg. por ciento). La depuración de creatinina variaba netre 86,5 y $143 \mathrm{ml}$. por minuto. El ácido úrico estaba elevado hasta $10 \mathrm{mg}$. por ciento.

Los autores consideran que el síndrome nefrótico es una complicación rara en el embarazo. Sus casos fueron tratados con corticosteroides.

\section{"El papel de las arterias espirales} en la patogenia de la pre-eclampsia" ha sido estudiado por I. A. Brosens, W. B. Robertson y $H$. G. Dixon, de Lovaina, Bélgica. Hacen una revisión de su numerosa colección de biopsias del lecho placentario y de las piezas quirúrgicas de cesáreas-histerectomías en pacientes normotensas y en hipertensas. Confirman que en la preeclampsia el lecho placentario se caracteriza por adaptación deficiente de las arterias espirales, en comparación con lo que ocurre en el embarazo normal, ya que en aquélla los cambios afectan solamente los segmentos deciduales sin alcanzar los miometriales, que retienen sus paredes músculoelásticas y su diámetro normal de 200 micras, mientras que en el embarazo normal el diámetro arterial más allá del límite deciduo-miometrial es de 500 a 1.000 micras. Este desarrollo insuficiente de las arterias espirales en la pre-eclampsia las hace especialmente propensas a las perturbaciones hemorrágicas.

Una contribución importante en el aspecto genético es la de R. Toaff y $M$. R. Peyser de Tel-Aviv: "EPH-Gestosis en el segundo trimestre y aberración cromosómica del conceptus". Los autores han revisado 19 embarazos triploides (1) avanzados, que han obtenido de la literatura publicada. En 8 de 14 casos con datos vitlizables, se presentó una EPH-gestosis severa, en- tre las semanas 16: y 24․ En todos éstos la placenta era demasiado grande para el tiempo de embarazo y presentaba zonas extensas de degeneración molar. El embarazo triploide avanzado se considera como un ejem plo más de "hiperplacentosis", responsable de la toxemia pre-eclámptica, y la hiperplacentosis, más que un simple factor cuantitativo, es la expresión de una hiperactividad trofoblástica.

\section{Diagnóstico Diferencial}

En los trabajos orientados hacia el aspecto diagnóstico surgen nuevamente la diversidad de criterios y la dificultad de establecer una secuencia patogénica. En la asociación nefroesclerosis-enfermedad hipertensiva, qué es lo primario y qué lo secundario?

Este es otro motivo de controversia en la sesión de discusión final del Symposium, al lado del significado patogénico de la coagulación intravascular diseminada en la gestosis, haciendo énfasis en la asociación nefroesclerosis - embarazo - preeclampsia eclampsia - anemia hemolítica.

Uno de los trabajos más importantes en el tema de la nefroesclerosis fue el de M. R. Peyser, R. Toaff y A. Aviram, de Tel-Aviv: "Seguimiento de las mujeres nefroescleróticas diagnosticadas en el embarazo".

En 80 pacientes, después de embarazos complicados por severa EPHgestosis, se hicieron biopsias renales percutáneas. En 18 de ellas (cerca de 25 por ciento) el diagnóstico histológico fue nefroesclerosis. Trece fueron seguidas hasta por 7 años; 3 conti-

(1) Triploide, dentro del grupo de características poliploides, en que el número de cromosomas de las células somáticas es un múltiplo (mayor de 2) del número haploide, que es el de las células germinales. 
nuaban hipertensas 6 meses postpartum. Otras 7 se volvieron hipertensas persistentes más tarde. En 4 se demostró disminución del flujo sanguíneo renal (prueba de la sulfofenolftaleína). En 6 se encontró disminuída la depuración de la creatinina.

La nefroesclerosis puede anteceder al embarazo y coexistir con la gestosis. La enfermedad hipertensiva relacionada con la nefroesclerosis es progresiva y puede llevar a la hipertensión sostenida, con daño de la función renal. El embarazo hace que la enfermedad se revele clínicamente. En la mayoría de los casos la hipertensión desaparece después del parto, para luego reaparecer eventualmente como hipertensión sostenida. El diagnóstico precoz permite una mejor atención en futuros embarazos y puede favorecer la prevención de las complicaciones vasculares tardías.

Los estudios de citoquímica ofrecen posibilidades diagnósticas.

D. Weistein, W. Z. Polishuk, E. Sadovsky, Y. Z. Diamant y H. Zuckerman, del Hospital Universitario Hadassah, Jerusalén, en su comunicación "La fosfatasa alcalina leucocitaria en el diagnóstico diferencial de la hipertensión en el embarazo", señalan un ascenso sostenido de la actividad de esta enzima (FAL) durante el embarazo, alcanzando un pico en las sema-

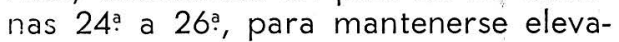
da hasta una semana antes del parto y descender después de éste bruscamente. En la gestosis los valores son generalmente inferiores a los normales (incluso varias semanas antes de que haya manifestaciones clínicas), pero no ocurre lo mismo en la hipertensión esencial, en la cual también son normales. En algunas pacientes la muerte fetal in útero estuvo precedi- da de un ascenso súbito a valores anormalmente altos, seguido de descenso. Este tipo de reacción es llamado por los autores "alarma FAL". Los resultados indican que el puntaje de FAL tiene valor en diagnóstico diferencial entre la hipertensión esencial y la gestosis.

La técnica empleada por Polishuk (Revue Francaise de Gynécologie et d'Obstétrique, 67: 115, 1972) consiste en tratar los leucocitos con fosfato de p-naftol y teñirlos después con hematoxilina. Se clasifican 100 leucocitos según una escala de puntaje que va de 0 a 4, para establecer un "índice FAL".

\section{Evaluación de la Madurez Fetal y de la Función de la Unidad Feto-Placentaria}

La investigación bioquímica es en este campo la orientación predominante, especialmente en el estudio de las enzimas, las substancias lípidas y las hormonas.

Y. Beyth e Y. Diamant, de la Universidad de Hadassah, Jerusalén, han estudiado "La actividad de las enzimas del metabolismo de los carbohidratos y los lípidos en la placenta humana, en la toxemia gravídica". Señalan un descenso significativo en la actividad de la kinasa pirúvica en todas las placentas toxémicas, en comparación con las normales, lo que puede indicar una reducción del potencial glicolítico en las primeras. En cuanto a diferencias entre la actividad enzimática correspondiente a los casos de fetos hipotróficos ("small for date") toxémicos y aquéllos fetos hipotróficos por otras causas ("small for date syndrome"), se encuentran las siguientes: 


\begin{tabular}{lcc}
\hline & $\begin{array}{c}\text { hipotrofia fetal } \\
\text { toxémica } \\
\text { KINASA PIRUVICA }\end{array}$ & $\begin{array}{c}\text { hipotrofia fetal } \\
\text { ottras causas } \\
\text { actividad } \\
\text { aumentada }\end{array}$ \\
$\begin{array}{l}\text { disminuida } \\
\text { (glucosa-6-fosfato } \\
\text { y }\end{array}$ & actividad & actividad \\
6-fosfo-gluconato) & disminuída & aumentada \\
\hline
\end{tabular}

Por su parte, M. Blum, F. Shabiay e I. Halbrecht, de la Universidad de Tel-Aviv, han estudiado la "Actividad de la leucina-amino-peptidasa sérica en embarazos normales, anormales y toxémicos": la actividad de esta enzima aumenta durante el desarrollo normal del embarazo. En embarazos patológicos es más baja que en embarazos normales. En la toxemia está considerablemente aumentada (como ocurre también en el daño hepático, lo que puede explicar este aumento en la toxemia).

S. O. Anteby, W. Z. Polishuk, Y. Stein y H. Bar-On, de la Universidad de Hadassah, Jerusalén ("Relación Lecitina-Esfingomielina en la Gestosis"), señalan las relaciones bien definidas entre los fosfolípidos activos del líquido amniótico y la integridad funcional del pulmón fetal. El cuociente lecitinaesfingomielina guarda estrecha relación con la edad gestacional, lo que dá a este índice valor cuando hay que interrumpir el embarazo en los casos de riesgo como la gestosis, pues ayuda a elegir el tiempo más oportuno del nacimiento, con miras a reducir el peligro de sindrome de dificultad respiratoria en el recién nacido, causa principal de la muerte neonatal. El método consiste en extraer los lípidos del líquido amniótico y hacer su determinación por cromatografía. Los resultados de la relación L/E fueron expresados en gráficas y se obtuvieron curvas correspondientes a 128 embarazos. Los datos preliminares indican que en la gestosis con insuficiencia placentaria hay una aceleración de la maduración pulmonar del feto, que se expresa por una más temprana obtención de cuocientes L/E superiores a los standards del embarazo normal. Lo contrario ocurre en la gestosis con diabetes, en que hay maduración pulmonar retardada. Puesto que el tiempo de terminación del embarazo depende de un balance entre la edad del feto y su situación de peligro in útero, se encaminan los esfuerzos hacia el hallazgo de parámetros múltiples que permitan establecer el tiempo óptimo para el nacimiento, en los casos de gestosis (1).

(1) Cfr. Obstetrics, Greenhill-Friedman, p. 173, Saunders, 1974

La importancia de la lecitina está relacionada con la evidencia creciente de que el síndrome de dificultad respiratoria del recién nacido tiene que ver con la madurez pulmonar del feto, más que con la edad gestacional, el peso al nacer 0 el modo de nacimiento. La madurez pulmonar parece guardar relación directa con la cantidad de lecitina en los pulmones y ésta se expresa en el contenido en el líquido amniótico. Los lípidos (lecitina y esfingomielina) se determinan por cromatografía. Según Gluck, estos dos son equivalentes antes de alcanzar la madurez pulmonar; después de la semana 35a. la Lecitina sube bruscamente, mientras que la Esfingomielina baja, de modo que el cuociente $L / M$ adquiere valores crecientes.

Cfr. Ob-Gyn Observer, 13: 8, 1974: En St. Margaret's Hospital, Boston, se ha desarrollado una prueba simplificada para cleterminar la lecitina en el líquido amniótico. Se basa en la degradación de la lecitina a lisolecitina por la enzima fosfo-lipasa-A. A su turno, la lisolecitina destruye los glóbulos rojos en una mezcla con etanol, líquido amniótico y solución salina. Si subsisten glóbulos rojos visibles, ello indica la ausencia de lecitina en el líquido amniótico. 
R. Kaufman, U. Eylat e Y. Abulafia, del Hospital Sharei Zedek, Jerusalén, han hecho un "Estudio comparativo de la hormona lactogénica placentaria humana, el estriol y la fosfatasa alcalina leucocitaria en embarazos patológicos". Los resultados indican que en la toxemia leve tratada los tres parámetros son inferiores a la mediana normal, siendo la HLPH la más baja. Esta se encuentra muy por debajo de lo normal en la toxemia grave y en casos no explicados de insuficiencia placentaria. Se muestra como la prueba más sensible para evaluar la función de la unidad feto-placentaria.

H. Yaffe, W. Z. Polishuk y E. Sadovsky (Universidad de Hadassah, Jerusalén) presentan una comunicación sobre la "Correlación entre el estriol urinario y los movimientos fetales en casos de toxemia y de fetos hipotróficos (small for date)". Los registros de movimientos fetales fueron correlacionados diariamente con determinaciones del estriol urinario en $16 \mathrm{em}$ barazos normales y 41 patológicos. Una disminución, seguida del cese de los movimientos, con ruidos cardíacos aún audibles, se define como "signo de alarma de los movimientos". Este signo se observó en 5 de 6 casos antes de la muerte fetal. Solamente en uno de ellos el estriol indicó una situación crítica.

En los casos de valores bajos o críticos del estriol, la monitoría diaria de los movimientos permitió la preservación del embarazo hasta lograr un feto más maduro, contra la norma convencional aceptada en estos casos, y puede considerarse como más significativa que el estriol para predecir la muerte fetal in útero.

El mismo grupo de autores en otra comunicación ("Significación clínica de los movimientos fetales en la gestosis") presenta 43 casos que incluyen pacientes de gestosis con o sin retardo del crecimiento fetal y pacientes con fetos hipotróficos sin causa aparente. Los movimientos fetales fueron contados en períodos de 12 horas, tanto por datos subjetivos de la madre como por monitoría electrónica. El "signo de alarma" (reducción o cese de los movimientos, con persistencia de ruidos cardíacos normales) se presentó en 15 casos, de los cuales 7 fetos murieron in útero y 8 nacieron vivos, vaginalmente o por cesárea. Se concluye que el signo de alarma indica perturbación metabólica y necesidad de interrumpir el embarazo, siempre que el feto sea viable.

M. Hermer y D. M. Serr, de la Universidad de Tel Aviv ("Evaluación cuantitativa de la rata de los movimientos fetales registrados electrónicamente en embarazos normales y toxémicos") estudiaron la "rata de movimientos fetales" (número de movimientos en una hora) mediante tres micrófonos fonocardiográficos colocados sobre el abdomen y con inscripción poligráfica, en 13 embarazadas normales, en 12 toxémicas y en 5 casos con fetos hipotróficos, todas ellas entre las 37 y las 41 semanas.

En las normales la rata varió entre 30 y 181, con una mediana de 90,1 en una hora; las pacientes percibieron un promedio de 37,6 por ciento de este número.

En las toxémicas la rata fue de 19 a 163, mediana de 91,6 por hora, y percibidas por la paciente el 35,2 por ciento.

En las de fetos hipotróficos, rata de 90 a 309, mediana de 163,6, y movimientos sentidos en 36,8 por ciento. 
Leo Tervila, de Lahti, Finlandia, ha hecho un estudio interesante sobre el desequilibrio metabólico del feto: "Influencia de la gestosis y de la labilidad tensional sobre el feto". Se tomaron micromuestras de sangre simultáneas de la oreja materna, el cuello uterino y el feto, en embarazadas normales, en hipertensas lábiles y en gestosis severas. Se determinaron los valores de P02, PH, BE y PC02. Los fetos no mostraban signos de asfixia. Las diferencias de los valores de BE entre las gestósicas y sus fetos fueron significativamente mayores que en los casos normales. No hubo diferencias de los valores P02, PH y PCO2 entre los dos grupos. Esto apoya la opinión de que en la gestosis el feto tiene tendencia a la acidosis metabólica, lo que lo sitúa en condiciones menos favorables si se presenta una hipoxia aguda. La P02 de los fetos de grávidas con presión lábil fue inferior a la de los fetos de madres normales.

\section{Tratamiento}

En una comunicación de I. MacGillivray, de la Universidad de Aberdeen ("Es nociva la pre-eclampsia leve para el feto?") se presentan conceptos interesantes basados en un estudio de mortalidad perinatal, peso fetal y retención hídrica, comparativamente entre primigrávidas normales, primigrávidas hipertensas y primigrávidas con proteinuria e hipertensión. Los resultados muestran que la retención de agua y la hipertensión no son necesariamente nocivos para el feto. El autor considera ante todo que una presión diastólica de 90 no es forzosamente anormal, aunque a veces pueda serlo, sino apenas una respuesta normal al embarazo, y que no afecta al niño, como lo muestran los datos del peso al nacimiento. Afirma que cuanto más peso gana la madre, mayor es el peso del niño. Considera como ganancia alta la superior a $1 \frac{1}{4} \mathrm{lb}$. por semana (570 gm.), y como ganancia baja la inferior a $3 / 4$ de lb. (340 gm.). En las pacientes con ganancia alta, aparece edema en el 70 por ciento. En las normales, en 38 por ciento. En las de ganancia baja, sólo en el 10 por ciento. Los diuréticos administrados después de la 30 a semana de embarazo son efectivos para reducir el peso del niño al nacimiento, pero no se sabe si son nocivos. La pre-eclampsia no se puede prevenir con diuréticos. For consiguiente, lo recomendable es no dar diuréticos.

En un trabajo de "Comparación entre el régimen hiposódico-diurético y el régimen libre no diurético", R. Borenstein, M. Lancet e I. Bar-Haim, de la Universidad de Hadassah, Jerusalén, discuten el tratamiento tradicional de la gestosis por limitación de la sal en la dieta y la administración liberal de diuréticos y señalan la inutilidad y el posible daño de este régimen. No obstante, teniendo en cuenta que los resultados clínicos con el método viejo han sido buenos, tanto para la prevención como para el tratamiento curativo, los autores decidieron comparar los dos métodos. Se informa en este estudio sobre un ensayo preliminar con 110 pacientes de las cuales 80 recibieron diuréticos y dieta hiposódica y las 30 restantes no fueron sometidas a estas medidas. En los dos grupos se recomendó el reposo en cama y se trató la hipertensión con drogas hipotensoras similares, si estaban justificadas. El diagnóstico de gestosis se basó en los criterios usuales de edema, proteinuria e hipertensión. La vigilancia diaria incluyó tensión arterial, peso, balance líquido y albuminuria cuantitativa. Periódicamente se examinaron la hemoglobina, el ácido úrico sérico, la creatinina sérica y la proteinemia. Entre los dos grupos no se observó diferencia en el 
curso de la enfermedad ni en el resultado. Los autores piensan que la omisión de los diuréticos y el empleo de una dieta normal no agravan la toxemia.

Un estudio conjunto de los Departamentos de Gineco-Obstetricia, Nefrología y Hematología de la Escuela de Medicina de Haifa, es presentado por J. M. Brandes bajo el título "Control de la pre-eclampsia severa por el tratamiento hipotensor agresivo solo 0 combinado con la heparinización crónica". De Febrero a Julio de 1974 se presentaron 17 casos de pre-eclampsia grave y dos de eclampsia, o sea una tasa global de 0,9 por ciento sobre el total de casos obstétricos. Las 17 pre-eclampsias se dividieron en dos grupos:

a) 10 casos de pre-eclampsia grave (con hipertensión severa) sin complicaciones fueron tratados con lo que los autores Ilaman "terapia hipotensora agresiva", en un esfuerzo por reducir la tensión arterial a menos de 140/90, mediante drogas solas o en diversas combinaciones, a saber: $\mathrm{Al}$ domin, Hidralazina, Deralin, Reserpina, Esbetal, Sulfato de magnesio, Valium. Las dosis dependieron de las respuestas.

b) 7 casos de pre-eclampsia presentaban las siguientes complicaciones:

1. Insuficiencia placentaria, diagnosticada por útero pequeño para la fecha y valores bajos de estriol y de H.L.P.H.

2. Compromiso renal grave: disminución de la depuración de creatinina (C.C.T.), valor inicial de $70 \mathrm{ml} / \mathrm{min}$.
3. Fruebas hematológicas de coagulación intravascular diseminada, es decir, valores altos de productos de degradación de la fibrina, o bajos del recuento de plaquetas.

En todos estos subgrupos se consideró posible la presencia de coagulación intravascular diseminada, y por consiguiente, además de la terapia hipotensora agresiva, se usó la heparinización crónica, administrando 12.000 a 36.000 unidades diarias de heparina por vía intravenosa, por varios días, a veces hasta por 5 semanas (1).

La decisión final de interrupción del embarazo antes de la semana 36 se basó en la relación L/E (lecitina/ esfingomielina) y la creatinina del líquido amniótico; si estos datos no mostraban una madurez fetal satisfactoria se continuaba el tratamiento hasta lograrla. En dos casos fue necesario terminar el embarazo antes de la madurez, por enfermedad incontrolable.

El cuidado de las pacientes fue como sigue:

Hospitalización en la unidad de alto riesgo, monitoría continua de la tensión arterial, pruebas de depuración de creatinina y determinación del estriol cada 48 horas, pruebas diarias de coagulación cuando se usó heparina. Las decisiones de tratamiento se tomaron en interconsulta (nefrolo-

(1) Cfr. Manual Merck: Dosis inicial de heparina 100 a 150 unidades por kg., seguidas de 75 a $125 \mathrm{u} / \mathrm{kg}$. cada 4 horas. Tiempo de coagulación (Lee \& White) una vez al día, tres y media horas después de una inyección, manteniendo la coagulación entre 20 y 30 minutos. 
gía, hematología y obstetricia), repitiendo las consultas de control dos veces por semana o con mayor frecuencia segúún la necesidad.

Las conclusiones fueron las siguientes:

a) Hipertensión. Con el régimen hipotensor agresivo se logró estabilizar la tensión arterial, aunque en los casos severos fue muy difícil lograr una reducción significativa de la presión diastólica. En tales pacientes hay que contentarse con prevenir un mayor ascenso.

b) La función renal se conservó o se mejoró con la terapia hipotensora sola, o combinada con la heparinización. c) Durante la heparinización crónica no se observaron complicaciones maternas ni fetales. No hubo complicaciones ni muertes fetales durante el parto. En dos casos se presentó tromboflebitis profunda 3 semanas postpartum.

d) En los 17 casos sólo ocurrieron 2 muertes fetales ante-partum.

e) No se presentó eclampsia en ninguna de las 17 pacientes de preeclampsia. Los dos casos de eclampsia de ese período habían ingresado ya con convulsiones.

f) No hubo muertes neonatales.

g) No hubo muertes maternas. 